\title{
Infection and atherosclerosis: is this hypothesis still alive?
}

\author{
Enrique Gurfinkel
}

Throughout the evolution of humans, the species has managed to survive by tolerating hunger, leading to the development of a peculiarly equipped endocrine system, and by having an immune system that adapts after each infectious episode. These two systems have allowed humans to evolve biologically through eras such as the Paleolithic and Neolithic ages, and into the modern era. The consumption of domesticated animals allowed humans to adopt a sedentary lifestyle and increase the intake of calories and saturated fats to levels far exceeding those of primitive hominids. Thus, access to the necessities for subsistence was facilitated without the former hunter-gatherer workload, and the species was able to quickly establish itself across the continents.

The endocrine and immune systems, which were developed to feed and defend the human organism, seem to have been affected by human cultural changes, and the atherosclerotic process has evolved in parallel. Essentially, though, survival's needs do not seem to have led to major biological changes. In truth, the human genome has mutated a mere $0.5 \%$ for every 106 years of evolution. By contrast, the genomes of the bacteria and viruses that challenge humans have mutated much more rapidly in order to survive over generations. For this reason, infection has always been a serious threat to humans.

Given the constantly changing characteristics of infectious agents, the presence of multiple antigens against different infectious structures in human blood is perhaps not surprising; many other antigens reside in vascular tissues, such as in the form of lymphocytes and macrophage cells, for example in atherosclerotic plaques. This coexistence is chronic, but the immune system's adaptiveness is weakened in the presence of powerful antigens; on the other ....an answer is required about whether the atherogenic process is triggered, accelerated or both by infection...

Enrique Gurfinkel is an Advisory Board member of Nature Clinical Practice Cardiovascular Medicine.

\section{Competing interests}

The author declared he has no competing interests.

www.nature.com/clinicalpractice doi:10.1038/ncpcardio0428 hand, the sudden appearance of attenuated antigens, such as in vaccination, strengthens the host's immune system. Thus, thanks to the development of antibiotics and the generalized administration of vaccinations, humans have become able to prolong life. Given this knowledge, an answer is required about whether the atherogenic process is triggered, accelerated or both by infection, and thereby the presence of powerful antigens, or whether it is an isolated and separate event.

The dominance of inductive scientific theory in practically all sciences means that the classic clinical study model has not been overcome: in the presence of an infectious agent, let us apply an anti-infectious agent to achieve the desired results. Results thus far have, therefore, been inconsistent. The fact that studies have yielded results leading in divergent directions indicates that the study designs are not comparable, and consequently it is not possible to draw clear conclusions about whether a relationship exists between infection and atherosclerosis.

In the face of this pathophysiologic situation, the investigative model should be modified. Perhaps this aim will be facilitated by advancing imaging techniques, which will enable the observation of temporal alterations in infected atherogenic plaques by assessment before and after treatment for infections. A recommendation by the WHO supports the need for such investigations. They now recommend that all individuals older than 50 years-down from 65 years-should be vaccinated against influenza. The reason behind this change is the marked reduction in morbidity across all subgroups of patients, particularly those with cardiovascular diseases. In my judgment, the hypothesis of a relationship between infection and atherosclerosis is still alive. 